\title{
Conducting Assessments in Technology Needs
}

\section{From Assessment to Implementation}

\author{
Cheryl A. Wissick \\ University of South Carolina \\ J. Emmett Gardner \\ University of Oklahoma
}

\begin{abstract}
Practitioners with an expertise in assistive technology and technology assessments are in demand to be full participants in the selection, planning, and implementation of instruction for students with mild disabilities. Frequently, practitioners with knowledge of assistive technology are assigned to evaluate students with sensory, physical, language, or severe disabilities. Our article highlights aspects of technology assessments and progress monitoring that can be used for students with mild disabilities. Given the impact that technology integration can have on the access that students with mild disabilities have to the general education classroom, we argue that all practitioners should be cognizant of protocols for assistive technology assessment and evaluation and that all evaluation teams should include an assistive technology specialist.
\end{abstract}

Keywords: assistive technology; assessment; evaluation

$\mathrm{T}_{\mathrm{s}}^{\mathrm{h}}$ he use of technology has been shown to be effective in a wide range of content areas (e.g., Ashton, 2005; Edyburn, 2004; Okolo, Cavalier, Ferretti, \& MacArthur, 2000). The No Child Left Behind Act of 2001 (NCLB) has challenged educators to ensure that all [emphasis added] students are provided with access to and the ability to make progress within general education standards. At the same time, the Individuals with Disabilities Education Improvement Act of 2004 (IDEIA) mandated that consideration of assistive technology (AT) devices and services had to be performed during Individualized Education Program (IEP) meetings. Therefore, an affiliated challenge facing special and general educators is to ensure that the integration of technology provides opportunities and supports for students with disabilities in general education classrooms. One consistent theme across much of the special education technology literature is that if technology is to be effective, practitioners need to consider how to match individual student needs with specific technology applications. For example, a particular software application or assistive device will not be appropriate or provide maximum opportunities to all students who are sharing a classroom or school setting. Assessment of students' technology requires consideration and understanding of a variety of related factors.

To evaluate and recommend technology for students with multiple or developmental disabilities-students who use technology for augmentative communication or mobility-schools and districts will often establish assistive technology teams (e.g., Ball, Bilyeu, Prentice, \& Beukelman, 2005; Beukelman \& Mirenda, 1998; George, Schaff, \& Jeffs, 2005). Unfortunately, AT teams usually do not focus on the needs of students with mild disabilities, who often spend the majority of their time in the general education classroom. For a variety of reasons (e.g., funding, personnel resources, training, availability), the technology that students with mild disabilities might require comes under the domain of instructional technology services and not AT. However, it is critical that a wider range of technology options be considered for all students in the context of the NCLB and IDEIA mandates. For these reasons, we will focus on the use of AT for students with mild disabilities and consider how the process of assessment for technology needs should be integrated into the assessment cycle for screening, placement, educational planning, and progress monitoring.

\section{Definitions for Assessment and Technology}

The Individuals with Disabilities Education Improvement Act of 2004 continues the mandate that AT devices and

\footnotetext{
Authors' Note: Address correspondence to Cheryl A. Wissick, 235 Wardlaw-Education, University of South Carolina, Columbia, South Carolina 29208. E-mail: cwissick@sc.edu.
} 
services must be considered at the IEP meeting. The definition of AT devices is as follows:

$\$ 300.5$ Assistive technology device. Assistive technology device means any item, piece of equipment, or product system, whether acquired commercially off the shelf, modified, or customized, that is used to increase, maintain, or improve the functional capabilities of a child with a disability. The term does not include a medical device that surgically implanted, or the replacement of such device. (IDEIA, 20 U.S.C. § 1401(1))

This definition has an impact on the assessment of students' technology needs. AT can be defined simply as any or all technology. Any technology that can assist to increase, maintain, or improve a student's performance in the general education classroom could be considered AT. For example, an IEP team might suggest the use of a word-processing software program and not a "device" for a student. Subsequently, given the need to consider the effectiveness of this software tool, the team should also assess particular needs of the student and suggest special aspects that could be included in the word processor (e.g., Marino, Marino, \& Shaw, 2006; Wissick, 2005b).

Although the definitions of assistive technology device and service remained the same from the Individuals with Disabilities Education Act (IDEA) Amendments of 1997 to IDEIA, IDEIA added the reference/definition of a concept not originally included in the language of IDEA 1997 (but included in the language of the Assistive Technology Act of 1998), universal design (UD):

$\S 300.44$ Universal design. Universal design has the meaning given the term in section 3 of the Assistive Technology Act of 1998, as amended, 29 U.S.C. 3002. (IDEIA, 20 U.S.C. $§ 1401(35)$ )

The definition of universal design under the Assistive Technology Act of 1998 is as follows:

\section{SEC. 3. DEFINITIONS AND RULE.}

(a) DEFINITIONS-In this Act:

(17) UNIVERSAL DESIGN-The term "universal design" means a concept or philosophy for designing and delivering products and services that are usable by people with the widest possible range of functional capabilities, which include products and services that are directly usable (without requiring assistive technologies) and products and services that are made usable with assistive technologies. (105-394, S.2432)

Both of these definitions (AT and UD) challenge practitioners to consider what the AT will be "worth" to their students. Universal design poses challenges for practitioners to consider the barriers that might be inherent in any activity for a diverse group of students and to consider ways to enhance the learning for all students without the use of assistive technology. AT, or any technology, however, can also provide a platform to reduce barriers for all students to increase access to classroom content. In reality, AT and UD are compatible concepts, and related to practices that teachers and special education practitioners should consider jointly.

According to Harris (1997), all teachers need to consider two questions before developing any technologybased activities. Those same questions can be modified and applied to AT within the context of UD:

1. Will teachers' use of AT (and universal design) enable students to do something that they COULDN'T do before?

2. Will teacher's use of AT (and universal design) enable students to do something that they COULD do before, but better?

Therefore, we want to select AT that is "worth it" to students who need to have access to and make progress in the general education curriculum. This perspective is compatible with King's (1999) definition of assistive technology: "Assistive means helping, supporting, and aiding in accomplishing practical functions, tasks or purposes. . . . Technology means reliance on simple as well as potentially highly complex tools, devices, and equipment" (p. 14).

When students use technology to help, support, and/ or aid them to accomplish something that they could not do before, that application of technology is certainly "worth it" and is something teachers should aspire to provide for their students. However, the process of implementing technology effectively for students with mild disabilities is much more complicated than determining the relative worth of different types of assistive technology devices and applications.

In addition to understanding the definition and importance of AT, practitioners need to consider a model for assessment that includes technology. A typical model for student assessment would include screening, eligibility, curriculum planning, and progress monitoring. Although one might default to a model where AT might only be considered at the time of the IEP, AT can easily be assessed and considered in other phases of assessment. At the time of screening, technology supports or UD features that can benefit the whole class can be implemented. When an individual student's needs to access the curriculum and/or receive instructional support or accommodations are evaluated, technology should always be part of the planning. When technology is used, ongoing progress monitoring should include keeping track of technology effectiveness just as skills are monitored in reading or mathematics. 
Regrettably, practitioners are not always taught direct skills in technology assessment or progress monitoring for technology. Fortunately, many models and training packages exist for use by individuals, parents, or districts that provide technology assessment methods and considerations.

\section{Models of Technology Assessment}

School districts can benefit by having a plan or manual for assessing technology needs. This will not limit an IEP team in discussing technology needs, but it will provide them with a model for factors to consider and information to collect on individual students. Some technology assessment guides have been formally published and provide practitioners with manuals and protocols that are similar to other assessment measures. Whether a district chooses to adopt a published technology assessment guide will depend on its philosophy for AT implementation. To conduct a comprehensive assessment for technology integration, practitioners need to consider models that provide support throughout the assessment process. Locating frameworks or models for the consideration of assistive technology and enhancing the performance of students with mild disabilities are two aspects that are needed to provide students with access to the general education curriculum. Edyburn (2001) offered a review of 12 different models that provided a framework for technology use in special education. He described three clusters: assistive technology consideration, technology-enhanced performance, and developmental implementation.

The Assistive Technology Outcomes Measures (ATOMS) project, funded by the National Institute on Disability and Rehabilitation Research (NIDRR), provides access to an Informational Database of Assistive Technology Assessments (Edyburn \& Smith, 2004). Within the online database (Edyburn \& Smith, 2004), practitioners can review information on 43 different assessment tools and models. The tools are also divided into five categories: augmentative communication, seating and positioning, computer access, school and education, and other. Tools are reviewed by format, population, age, AT service area, and purpose (screening, referral, comprehensive assessment, matching person and technology, acquisition, implementation, follow-up, impact, or outcome). Practitioners can search the database for assessment tools and models that seem appropriate to their needs, access detailed information on the tool or model, and be provided with a URL for the publisher or link for online access to a relevant Web site. Before starting any assessment, practitioners would be advised to review this database to locate current information. It should be noted that Edyburn and Smith (2004) do not discuss every one of the 43 different technology assessment tools; they highlight only a few.
Given the focus of NCLB and IDEIA 2004, practitioners need to focus on models or possibly combinations of models or frameworks that can directly assist them in assessing their individual student's technology needs. Models developed entirely for adoption of augmentative communication, sensory supports, or mobility have not been included. All of the models reviewed have one aspect in common: They all focus first on the student or individual. For a practitioner or an IEP team to consider technology, they must first consider the student as an individual with a disability, then they must examine how these strengths, weaknesses, and other student characteristics interact with any barriers that may exist in the student's environment. Determining which technology tools can be effectively used to enhance students' performance requires a systematic process well beyond simply equipping a classroom with a variety of technology devices or software products.

\section{HAAT Model}

Cook and Hussey (2002) discussed the HAAT model based on the interaction among three parts: the human, the activity, and the assistive technology. According to Cook and Hussey, holding all these parts together is the context in which the technology is used for persons with disabilities. For these parts to fit together, their interaction must be given equal weight. The activity is the goal to be achieved, and the human has the skills available to meet the goal. The context bringing these skills and goals together defines constraints on achieving goals. The assistive technology therefore provides an external way for the human to perform the activity.

Obviously, this is a simplified description of a complex process. The HAAT model is mentioned first because it serves as a foundation for certified AT specialists and providers. For practitioners wishing to become a certified AT specialist, a careful study of the Cook and Hussey (2002) model and text is essential. Although the HAAT model is heavily implanted in the rehabilitation science industry as the model to consider when developing new technologies for persons with more severe disabilities, it includes some key ideas that relate to assessment for students with mild disabilities. At the core of this model is the interaction between the student, the environment, the task to be completed, and then how the AT fits into that puzzle. This is a fundamental perspective that is found throughout many, if not all, of the other AT or special education technology assessment models.

\section{Essential Human Factors Model}

Although an analysis of King's (1999) text Assistive Technology: Essential Human Factors might indicate that 
the focus is only on individuals with sensory, language, or physical impairments, the factors discussed can be incorporated into many of the other technology assessment models. The Human Factors model is more of a framework about what practitioners need to think about when selecting technology than a model with forms and steps to follow for selection.

King (1999) devised a model that applied Baker's (1986) basic ergonomic equation, which considers students' motivation and load factors in deciding the usefulness of assistive technology. King expanded the model and defined successful AT use as the balance of an AT user's motivation to complete a task, divided by the combination of physical, cognitive, and linguistic efforts, plus the time load. For example, a high school student who takes a computer to class might not be motivated to use it daily if the computer is heavy and requires a long time to load and access files at the beginning of every class. In addition, practitioners might not be willing to support students' use of this technology if a significant segment of every student's learning and instruction time is wasted during every class period.

King's (1999) discussion on the use of technologies and human factors applies to a variety of areas necessary to be successful in everyday life. Several of these human factors are critical in selecting technology for students with mild disabilities, especially if practitioners want to choose technology that is "worth it" for students. According to King, technology must be transparent or translucent - it must be easy for the user to understand or easy for others to interpret the user's intent. Technology tools must not appear different or make users stand out in their environment. The sequences used to access the technology must follow a logical flow. Consider, for example, a new software program that includes totally different terms/commands to open, use, or print documents. Similar to this, do the devices work the way we think they should work or will students always be trying to push the handle in ways that appear intuitively wrong? Technology must foster success or users will move into a cycle of learned helplessness. If software is too difficult to learn, the user may feel that the technology is not worth the time and effort. The technology must be devised so that the user gets some feedback on its use. Finally, the underlying operation of the technology should be similar to other devices or tools the student has previously used. Consider a calculator with the keypad numbers in a reverse order from the other calculators that are used in the classroom. King's perspective on human factors serves as a baseline regarding the importance to consider these factors when taking a closer look at models of technology assessment, especially those that include specific forms and surveys to guide the assessment process.

\section{Functional Evaluation for Assistive Technology (FEAT) Assessment Model}

The FEAT model (Raskind \& Bryant, 2002) considers the use of technology in the context of learning difficulties that persist across the lifespan of individuals with disabilities. According to Raskind and Bryant,

\begin{abstract}
Selecting the [technology] device [or software] that will achieve the greatest efficacy for an individual with a learning problems requires careful analysis of (a) the interplay among the individual's specific strengths, limitation, special abilities, prior experience/knowledge [with AT], and interests; (b) the specific tasks/functions to be performed (e.g. compensating for reading, writing, or memory problem); (c) the specific contexts of interaction (across settings-school, home, work; and over time- over a semester or a lifetime); and (d) the specific device (e.g. reliability, operational ease, technical support, cost). (p. 5)
\end{abstract}

Of the group discussed in this article, the FEAT model is one of the more formal and systematic models of technology assessment for students with special needs. The FEAT is composed of five scales/checklists that are used as part of the assessment process to identify, select, and evaluate ways that technology can help a student access and make progress in the general education curriculum. Professionals with knowledge and skills regarding use of assessment scales, individual differences, cognitiveacademic interventions, and knowledge and skills regarding assistive technology fit the qualifications to serve as a FEAT examiner - the individual who coordinates the administration and interpretation of the FEAT scales. Typically the FEAT examiner is the head of the IEP (or Assistive Technology) team, while individual team members or other stakeholders-persons who are familiar with the students' abilities and/or curricular needs and/or technology supports-complete specific FEAT scales. Brief explanations of the individual FEAT scales follow.

Contextual Matching Inventory (CMI). The CMI assesses the frequency - whether it be daily, weekly or monthly_with which an individual may be expected to perform tasks that may benefit from AT support across different settings, relative to tasks that involve knowledge and skills, in the context of nine areas: listening, speaking, reading, writing, math, memory, organization, physical/motor, and social behavior. For example, one item on the CMI asks a teacher how frequently the student is required to read from a textbook or handout, whereas another asks how frequently the student must read computer text. 
Checklist of Strengths and Limitations (CSL). Assistive technology users may have a variety of cognitive and academic strengths that can be used as anchors for compensatory AT applications. The CSL reviews the strength from weak to strong of the individual who will be using AT, relative to abilities that are representative of the nine contextual areas of the CMI. For example, one item on the CSL rates the ability of the student to read words accurately; another item rates the student's ability to use appropriate vocabulary within written assignments.

Checklist of Technology Experiences (CTE). The CTE is used to assess the degree that the individual has had any prior experiences (from a lot to not appropriate) with a set of commonly used AT devices or AT software. All experience is related to the nine contextual areas of the CMI: listening, speaking, reading, writing, math, memory, organization, physical/motor, and social behavior. In the area of reading-related abilities, experiences with optical character recognition and/or text-to-speech would be recorded. For writing-related abilities, any experiences with a word processor or with word prediction or outlining programs would be noted.

Technology Characteristics Inventory (TCI). The TCI is used prior to formally introducing support technology, whether it be a device or software tool. Team members review every device or software tool and rate its characteristics based on a variety of factors, including its reliability, area of appropriateness to and compatibility with the individual and learning task, ease of use, level of manufacturer support, and ease of installation.

Individual-Technology Evaluation Scale (ITEC). Finally, the ITEC is completed for every device and software tool that has been implemented with the student. It assesses the factors that include, but are not limited to, the individual's use of technology relative to the technology's effectiveness; the student's interest, proficiency, comfort, attending, and degree of additional support needed while using the technology; and the extent to which the individual has been able to learn to use the technology in the context of academic/cognitive tasks.

Comprehensive Assessment. The FEAT scales concretely and objectively address a comprehensive range of factors associated with the technology applications as they relate to the instructional and learning needs of individuals. These scales measure the individual's abilities; specific academic, cognitive, and/or social tasks; the context over settings and time that these tasks occur; and specific technology devices or software products. The FEAT assessment can be very directed with administration in a structured/sequential format, or it can be flexible, depending on the context and need. As needed, only specific scales can be selected, and within each subscale, the items are extremely precise in how they characterize the factors. The FEAT does not ignore the physical or motor skills of the individuals; however, its focus clearly is attuned to assessing technology for supporting the learning and performance needs of the individuals relative to the day-to-day academic/cognitive aspects of their learning and their need to access the curriculum to perform typical school/learning activities. The domains assessed on the scales are straightforward; the items are directed at gathering information relative to specific needs.

\section{Matching Person With Technology (MPT) Model}

The MPT model and assessment instruments (Scherer, 1998, 2005a) were developed to focus attention on the needs and the preferences of the user, the aspects of the environments in which the technology will be used, and the functions and features of the technology. The focus on the MPT is to create a quality match between the user and the technology so that the technology will be used optimally. The MPT has been shown to be effective in helping users and caregivers organize these factors that might affect AT use. It consists of instruments that have been validated for use by persons with disabilities age 15 years and up, and another version has been developed to target technology use by infants and children. With the focus on older students, the MPT provides an approach for technology assessment in the context of high school students and transition teams.

Sample forms may be viewed at http://members.aol .com/IMPT97/MPT.html and are available on a CD-ROM (Scherer, 2005b) from the Institute for Matching Person and Technology. These are paper-and-pencil forms that may be completed in an interview format with the user. The process can take from $15 \mathrm{~min}$ to $45 \mathrm{~min}$, depending on the comprehensiveness of the evaluation. Scherer designed the MPT model to be completed as an active, presentoriented process, not one in which an examinee's responses are gathered for a team to review at a future meeting. The MPT follows a relatively straightforward, three-step process:

1. assessing the individual's technology use,

2. identifying appropriate technology, and

3. monitoring the individual's progress using the identified technologies.

Assessing Technology Use. The first assessment in the MPT is a preassessment survey of technology use, thereby identifying technologies with which an individual feels comfortable, as well as any possible negative influences of prior technology use. For example, high school students who have used word prediction or voice 
recognition in junior high with little success may be reluctant to try these tools again. Practitioners who recommend technology to support the more intensive demands of writing at high school (or college) would thereby need to take into account some individualized strategies for motivation and instruction.

Identifying Appropriate Technology. After the preassessment survey, the practitioner would use MPTprovided forms to assist in the identification of appropriate technology. The most important of these is the Assistive Technology Device Predisposition Assessment (ATDPA) to help the practitioner and individual select assistive technologies. The development and validation of the ATDPA followed steps for test/measure construction that included concept definition, draft of items, pilot testing, and determination of measure quality.

After completing the initial survey, the user and the practitioners begin by using the ATDPA to write down both goals that they have established together and possible alternative goals. This step in the process would be especially useful for transition plans. Based on each goal, possible interventions that are supportive of the goals are suggested, and specific technologies that can help the individual reach the goals are listed. Additional forms in the MPT include the Educational Technology Predisposition Assessment (ETPA) and the Workplace Technology Predisposition Assessment (WTPA). All of these forms serve as a means to identify appropriate technology for a person when considering his or her needs and goals, barriers that may exist to technology use, areas to target for training for optimal use, and type of additional support that may enhance use.

Monitoring Progress. An important part of the MPT model involves the continual reassessment of the individual's use of technology. After the person has received the most appropriate technology for his or her use, the MPT forms can be readministered one or more times to assess changes in the user's capabilities, the overall quality of life, and factors such as mood and social participation and support.

\section{Human Function Model}

Blackhurst (2001) based his description of the Human Function model on the premise that when considering AT, the focus is not the disability but rather the student's ability to function in a particular environment. A student encounters an environmental demand to perform some function or activity that he or she has difficulty performing due to the circumstances or particular capacity of a specific situation. In this model, function is therefore defined as the action a person takes to meet a demand. With respect to technology assessment for persons with disabilities, when viewing the process of AT selection, the staff at the National Assistive Technology Research Institute (NATRI, 2006) categorized seven functions within each context of school, community, and home.

The Human Function model of assistive technology is viewed from a larger perspective than just educational. Lifelong functions include existence, communication, body support, travel and mobility, environmental interactions, and recreation. Again, for the purposes of this review, which focuses on students with mild cognitive disabilities, only the areas of education and transition will be discussed in depth.

Blackhurst (2001) developed a graphical model that provides practitioners with a flowchart for decision making (see http://natri.uky.edu/resources/reports/function .html). Within this model, the functional demands of the task are considered, along with awareness of external supports and personal perceptions and characteristics. These aspects come together to form the basis for assessment, experimentation, and possibly adaptation. Once choices, which may include technology, are made, evaluation of the functional response and data on personal changes are collected. Personal changes might occur in the areas of strengths, achievement, independence, control, development, or coping strategies. Finally, a new cycle of evaluation and feedback is implemented.

This model has been incorporated in the Assistive Technology Planner: From IEP Consideration to Classroom Implementation (NATRI, 2006). Research on the development and use of the AT planner was sponsored by the U.S. Office of Special Education Programs. (An AT Technology Implementation Plan form can be downloaded from the NATRI site [http:// natri.uky.edu/atPlannermenu.html].) The planning form assists teams in monitoring and planning for AT implementation. This form contains areas to list the implementation team by name and role, AT equipment by name and status, equipment tasks with due dates, training needed, and plans for classroom and home implementation with notes related to IEP goals. Finally, there is a section for monitoring results and tracking progress.

For teams who need more detailed guides for the actual assessment, the UKAT Toolkit (Lahm \& Case, 2003) also incorporates the human function model and hosts free downloads available at http://edsrc.coe.uky.edu/www/ ukatii. Forms include Consideration, Pre-Assessment Profile, Assessment Planning and Data Collection, Assessment Report Outline, Trial Implementation, and Assistive Technology Implementation. The Consideration form includes a space for the team to note difficulties in any of the seven areas of human functioning. As the UKAT Toolkit incorporates the human function model, these forms make an excellent supplement to the AT Planner. 


\section{SETT Framework}

Zabala (1995, 2002) developed and revisited a framework for considering students and instruction. The SETT framework has wide appeal because it is intuitive and can be adapted for individual district needs. Of all the models described in this article, it probably is the one most widely implemented. The SETT framework focuses on key questions to guide teams in considering the student, the environment, the tasks, and the tools or the technology. Zabala stressed that SETT is designed to function as framework. It is not a protocol, involves collaboration and communication among team members who bring multiple perspectives on the student and the environment. The SETT framework provides practitioners with guiding questions to consider assistive technology. Practitioners need to consider the student's abilities, needs, and type of performance required. With the environment in mind, team members discuss the context, arrangement, and supports available to the student and the people supporting the student. Next, they ask questions about the tasks, to consider whether accommodations can be made to the activities. Finally, they consider the types of tools (technology or others that provide functional support) that may reduce the barriers to participation in the tasks. Zabala $(1995,2002)$ urged teams to use the SETT framework in a circular manner, consistently reevaluating the student within different environments and tasks.

Zabala $(1995,2002)$ has also developed a form for teams to take notes on the assistive technology assessment process. This form is available from the Assistive Technology Training Online (ATTO) Web site (http:// atto.buffalo.edu/registered/ATBasics/Foundation/Assess ment/sett.php) in the section on AT basics and AT assessment. ATTO presents the SETT framework and provides links to forms from other AT centers that support the SETT framework.

Although Zabala $(1995,2002)$ developed SETT as a framework for AT teams, a variety of organizations, agencies, and authors have developed materials that apply to and/or compliment the model. The Wisconsin Assistive Technology Initiative (WATI) has developed forms/guides for gathering student information and environmental observations that apply to the SETT framework. Bowser and Reed (1998) supplied a complete listing of educational checkpoints for technology noted on the WATI Web site (http://www.wati.org), and they have also developed a student version of this model, titled Hey, Can I Try That? (Bowser \& Reed, 2001). The Georgia Project for Assistive Technology (GPAT; 2006) has a consideration checklist for teams to consider the student's tasks, what the student can do independently, and what accommodations might be needed. For a listing of specific tools to consider, the resources section of the GPAT Web site provides an information guide with types of technology tools related to tasks. Finally, Zabala worked with the Texas Assistive Technology Network (TATN) to develop complete training modules and supporting forms. Six comprehensive modules are available at the TATN Web site (Texas Assistive Technology Network, 2006). The Consideration module was developed to train teachers about assistive technology and to provide an introduction to a SETT process for assessment. This module includes presenter and participant guides. The implementation module was developed for practitioners involved in the AT decision making and implementation and includes presenter materials with slides, notes, and demonstration forms. For the participant, there is a module that contains note-taking guides, a booklet of handouts, and blank forms.

\section{IMPACT2 Model}

Smith (2005) described the IMPACT2: Integrated MultiIntervention Paradigm for Assessment and Application of Concurrent Treatments model as representing a comprehensive evaluation process that includes preintervention, context, baseline, intervention approaches, outcome covariates, and outcomes. Perhaps the most inclusive of the models, IMPACT2 focuses on AT from preevaluation to final outcomes and is designed for practitioners who require a comprehensive look at all the aspects of technology assessment and implementation. In preintervention, school systems develop a curriculum based on universal design for learning principles to prepare classrooms for diversity. If students encounter difficulties working with the universally designed curriculum, the context has to be evaluated. Practitioners focus on the student and his or her needs, considered within the context of the environment and the tasks to be performed. This model incorporates features of the SETT framework. This preintervention phase results in a baseline measure that includes a functional assessment of the task/context and/or a measure of the performance quality of the student with the universally designed curriculum. At this point, most models for technology assessment stop, but in the case of the IMPACT2 model, this is only the first phase of the total technology-related evaluation.

During intervention, assistive technology is considered as one of the solutions; however, other options are considered that include solutions to reduce the impairment, compensate for the impairment, redesign the activity, redesign the environment, or provide a personal assistant-using technology-based or nontechnology-based methods. Each of these options is considered in the context of funding, and personal satisfaction is also considered a covariate of the final 


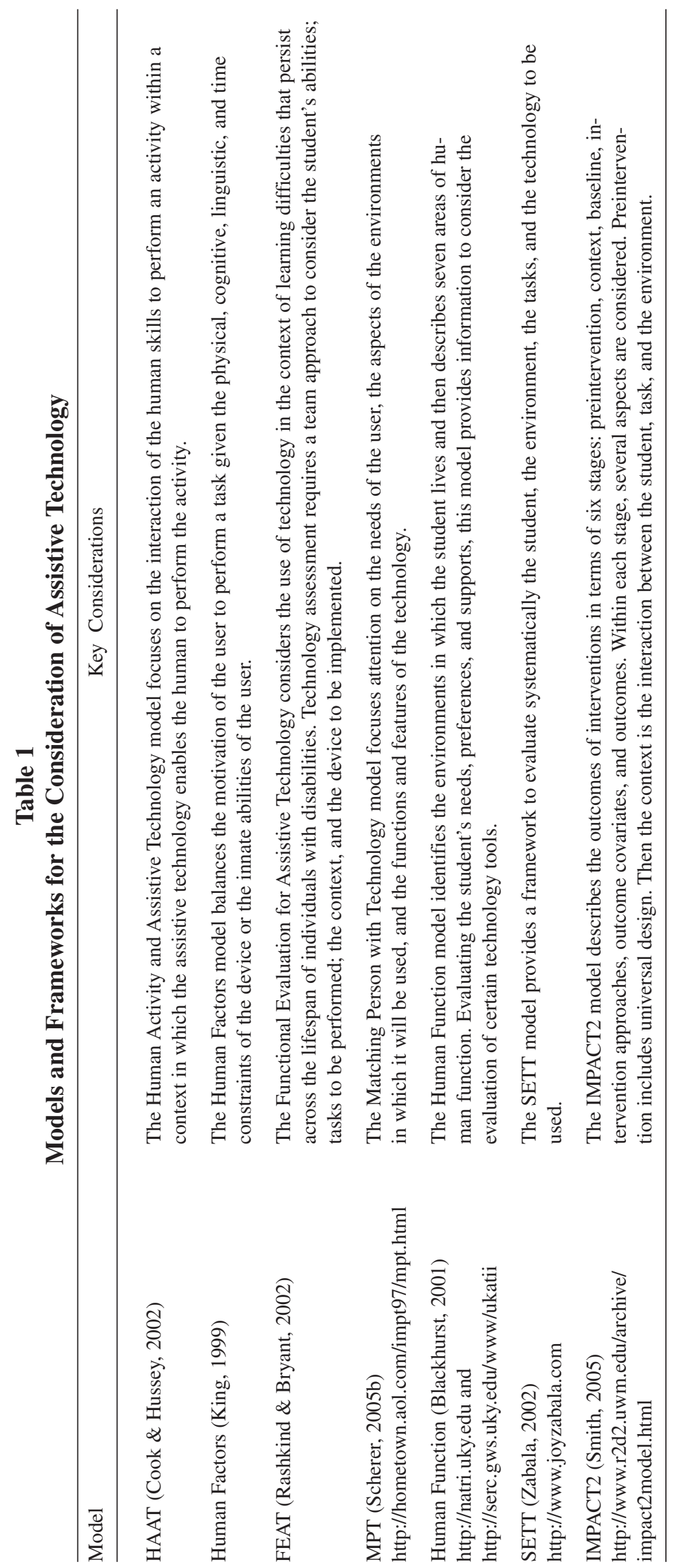


Figure 1

Model for Functional Assistive Technology Assessment: Alternative Pathways to Student Access

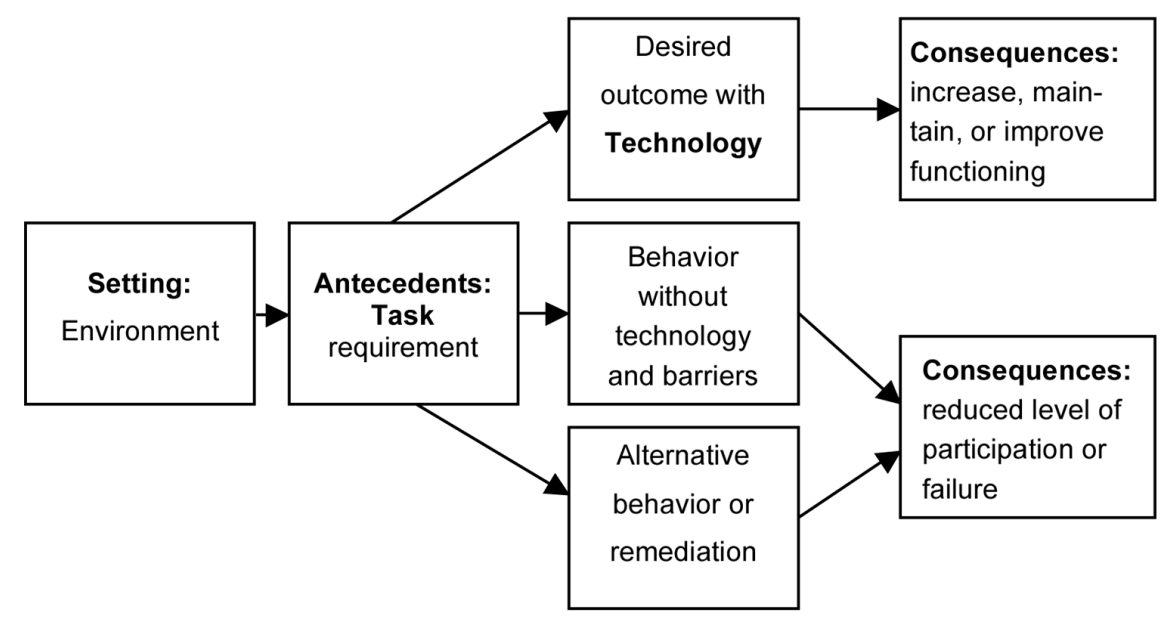

Source: O’Neill et al. (1997).

outcomes that will lead to use or nonuse of the intervention. The final outcome is thereby reached as a function of improved performance and quality of life. The ATOMS Web site (http://www.r2d2.uwm.edu/atoms) contains information and a detailed PowerPoint presentation on this model. Table 1 provides an alphabetical listing of the seven models that we discussed and provides key considerations for practitioners when assessing technology needs for students with mild disabilities.

\section{Comparing Models and Frameworks}

Conducting assessment for the technology needs of students with mild disabilities parallels many of the strategies practitioners use to identify problem behaviors in the classroom. Figure 1 depicts a simple but functional assistive technology assessment model based on the Competing Pathways model of functional assessment of problem behavior (O’Neill et al., 1997). Applying a functional approach to the use of technology, practitioners consider student behavior in the context of the setting, the antecedent (the requirements of the task the student must perform), and the barriers to completing the task. Without support, the student is unable to function at a level commensurate with his or her true potential and abilities, and the logical (sometimes only) other options are to modify the task or lower expectations. Rather than lower expectations, the prudent functional approach model is one in which desired outcomes using technology supports are determined via valid assessment procedures, resulting in the student being able to increase, maintain, or improve his or her behaviors.
All the models reviewed in this article can be simplified and graphically represented with this model, as there are consistencies in the key considerations. The focus is first on the student's skills or perceptions, to develop a picture of his or her strengths and weaknesses. Once practitioners have evaluated the student, barriers to instruction and the context for technology use are considered. The last task is to review types of technology that are appropriate for the task, selecting those that are most likely to result in positive functional consequences and outcomes. Unfortunately, many practitioners and parents are very familiar with the student and the context for technology use but are not always familiar with options available for integration within the general education classroom. At this point, and as a result of the technology assessment, additional resources or training might be required to successfully implement decisions arrived at through the technology assessment process.

\section{Resources for Technology Acquisition}

After considering all the needs, IEP teams and practitioners are challenged to locate an appropriate device or software application that would best meet the needs of the student. Unfortunately, due to the cost of specialty programs and devices, practitioners cannot afford a trialand-error method of choice. Being aware of free tools and downloads can assist in gathering data on response and training issues. Wissick (2005a) developed a Web toolbox linking and describing free technology tools to support basic skills (http://www.ed.sc.edu/caw/toolboxfree.html). 
In addition, many software companies provide 30- to 90-day free trials of software. From the Web toolbox on free tools, there are links to additional resources for free trials. Even 30 days is enough time to collect baseline data on the appropriateness of a tool or software application. For an overview of devices and application, Ability Hub (http:// www.abilityhub.com) features definitions and links to vendors.

If neither free tools nor downloads are available, visiting a local or state Assistive Technology Resource Center might be appropriate. A list of centers that are funded by the by Assistive Technology Act of 2004 can be found at the Rehabilitation Engineering and Assistive Technology Society of North American (RESNA) Web site (http:// www.resna.org/taproject/at/statecontacts.html). Wissick and Schweder (2006) reviewed several AT centers and provided information on resources and services. Many of the AT centers provide manuals, planning forms, and student user guides. Two other online organizations provide information on instructional technology tools to support students with mild disabilities. LD Online (http:// www.ldonline.org) provides a listing of and selected articles on different tools. Schwab Learning (http://www schwablearning.org) presents an online guide and a planning form in PDF format for parents and teachers to use in selecting technology. The model used in Schwab Learning's guide is similar to the models we previously presented, focusing on the interactions among the individual, context, tasks, and technology. Parents can also access related articles on consumer tips for selecting and evaluating technology. Specific AT centers and online organizations that provide free downloads and assessment protocols are listed in Table 2, and links can be found online at http://www.ed.sc.edu/caw/atrctoolbox.html.

What is important to consider at this step of a student technology needs assessment is that educational planning focusing on AT is not, and will not be, a stand-alone application. Students need to be trained on the use of the device or software application within the appropriate environment. Practitioners cannot assume that students with mild disabilities will learn to use the tool independently or that they will continue to use it across time and conditions. Assessment for education planning involving technology is critical, and objectives and ongoing assessment for using technology tools need to be incorporated into students' IEPs.

Because AT can also be included on an IEP for accommodations or modifications for high-stakes assessment, practitioners must be mindful that these tools must be used consistently in the classroom. All accommodations or modifications listed on an IEP for high-stakes assessment must be included as part of daily classroom assessment (Johnstone, Altman, Thurlow, \& Thompson, 2006). For example, if a student is to receive oral computer-generated administration of a test, all classroom tests should also be administered in the same manner. Unfortunately, the research on effectiveness of technology accommodations for high-stakes assessment is equivocal (Coughenour, 2005; Johnstone et al., 2006). At some point in time, practitioners may need to shift the assessment focus from identifying and selecting AT, to instruction and continual progress monitoring of the use of AT.

\section{Instruction and Progress Monitoring}

Once a student has access to a technology application or device, practitioners must be prepared to move to another phase of assessment. First and foremost, teachers have to be trained on the software or device to implement it effectively with the student. Second, they have to be able to train and offer direct support to the student during the implementation phase. This process is similar to educational planning: Goals and objectives are developed for the student, and then the student is provided direct instruction to master the goals.

To be successful in using technology to provide direct instruction and to help master learning goals, teachers and practitioners may need to meet to obtain additional training or share knowledge regarding technology and related resources across classrooms or schools. Local software representatives may need to provide direct training for comprehensive products. For example, once a student has been trained to use a new technology tool or software, human factors once again must be considered. The student must be motivated to use the technology application relative to successfully completing a task. As stated earlier, this process must be "worth it" to the student. Teachers or practitioners will need to employ a variety of direct instruction and positive behavior support methodologies to assist in making a smooth transition. Implementing technology at this stage is not really different than starting a new unit in social studies. It should be expected that students with mild disabilities will exhibit all of the same patterns-both adaptive and maladaptive learning and behavior patterns - that they exhibit when learning any new and unfamiliar content. Good teaching requires that educators anticipate events that may also affect students' stability, such as medication changes, schedule changes, illness, or unusual transitions. When any of these factors 


\begin{tabular}{ll}
\hline $\begin{array}{l}\text { Assistive } \\
\text { technology } \\
\text { Assessment }\end{array}$ & \multicolumn{1}{c}{ Web Site } \\
\hline ATOMS & $\begin{array}{l}\text { http://www.r2d2.uwm.edu/ } \\
\text { atoms }\end{array}$ \\
ATTO & http://atto.buffalo.edu \\
Boston Access & $\begin{array}{l}\text { http://boston.k12.ma.us/teach/ } \\
\text { technology/access.asp }\end{array}$ \\
GPAT & http://www.gpat.org \\
UKAT & http://serc.gws.uky.edu/www/ \\
ukatii \\
NATRI
\end{tabular}

\begin{abstract}
Assistive Technology Outcomes: Informational database of AT assessments; graphic model of the IMPACT2 model
\end{abstract}
Assistive Technology Training Online: Handouts and tutorials on software use; AT process guide and forms based on SETT model Student Access Map (SAM) model planning form to consider standards, stu-
dent, barriers, and tools
Georgia Project for Assistive Technology: Videos, handouts, and charts with defi- nitions on types of devices
University of Kentucky Assistive Technology Toolkit: Complete manual for assistive technology
National Assistive Technology Research Institute: Information on the AT Planner Implementation Guide

Texas Assistive Technology Network: Complete implementation training module with training guide and forms based on the SETT framework

Specific information on tools for students with learning disabilities

Rehabilitation Engineering and Assistive Technology Society of North America: List of Tech Act Funded AT centers

\begin{abstract}
Setting the Stage for Success: Detailed information on the SETT framework and on the quality indicators for assistive technology implementation (QIAT)
\end{abstract}

Technology and Media Division of Council for Exceptional Children: Resource for AT Planner and monographs on AT implementation

Wisconsin Assistive Technology Initiative Free download of "Hey Can I Try That?" and information on Education Tech Points continues for a long period of time, and the technology is no longer being implemented, a reevaluation needs to occur. Just as in functional assessment of problem behavior, if the problem keeps occurring, reevaluation of the student within the setting of the tasks is required. Of importance, then, is how to monitor the use of the tool and the types of data that need to be collected.

Zabala and Korsten (1999) have developed a protocol for monitoring the effectiveness of technology implementation. They suggested targeting specific areas in which change (e.g., learning or behavioral outcomes) may be expected. Any one of the following areas could be measured: quality or quantity (of learning), and behavioral factors such as changes in the technology users' independence, accuracy, frequency, spontaneity, or participation. In discussing these "quality" indicators for evaluation of effectiveness, the QIAT Consortium Leadership Team
(Zabala et al., 2000), stressed objective measurement of changes. Teams should decide what behavior(s) need to be measured, make a projection for when behavioral goals will be reached, and decide who should be responsible for data collection.

Discrete measures of specific outcomes are important, because without them, practitioners may assume that technology is not making a difference. For example, if a student starts to use a word processor, the immediate effect may be that the rate and quantity of writing decreases. From one perspective, this implementation of the technology may not be considered successful. In contrast, if accuracy and quality of writing-related behaviors are measured, rather than rate and quantity, it very possible that the student might actually have improved in desired behaviors as the result of the technology support. Taking this scenario a bit farther, if the student does increase in 
accuracy in the short term, continued use would be recommended. However, if after several months of using the technology the student still has not gained speed or increased quantity, the team might want to reevaluate the technology tool and make modifications.

Many protocols stress the importance of user attitudes and preferences for technology. Although this is important as a factor in initial motivation, many times students cannot accurately predict what tools would be best suited for them. A student might choose not to use a word processor but be able to do better quality work with one. Alternatively, some students may enjoy and want to use certain technology that has little effect on increasing their capabilities. In this case, practitioners may need to view technology as an asset that functionally increases or maintains students' motivation and skills.

To ensure success and increase motivation, students should be encouraged to track their own progress in the use of tools. For example, students using the wordprocessing software Microsoft Word can use the Word Count feature to take a daily measure of the number of words they have written. In addition, they can record the reading level or the readability of their work. The students can gain another technology skill by tracking their own progress on a spreadsheet and learning how to chart the progress by creating line or bar graphs. Many software tools may have built-in features that make it easier to collect data. For reading fluency, students could use the computer's clock to record beginning and ending times. Computers can be programmed to issue self-monitoring tones or visual prompts to assist students in maintaining attention to task.

Data regarding a student's mood and acceptance of the technology are important human factors to measure. Just as observation is used to document a student's interests in or need for the technology during assessment activities, direct observation of the student using technology in a general classroom and participating in class/curriculum activities can also be a valid way to document technology effectiveness. To assess specific information on functional relationships or consequences that may occur when a student uses technology within a particular learning environment, a classroom observation form could be adapted to collect information related to instructional/task conditions and different student behaviors (see Figure 2). Using time sampling procedures, data collected from forms like the one in Figure 2 can be used to compare, graphically or statistically, behavior changes over time and/or between different settings.

\section{Cautions and Final Thoughts}

Even after careful assessment of the student, environment, and selection of the technology tool, the implementation of technology can still fail. If practitioners work with parents and students to assess the need for assistive technology and also provide direct instruction on the use of the technology and monitor progress, implementation should be successful. Overall, the technology should provide the student with the supports to do something that he or she could not do before; therefore, it is not the purpose of our article to belabor reasons for failure that rest in the student. Instead, this section will highlight (a) points that we might have missed in the previous sections that might cause failure and (b) possible solutions that are under the control of practitioners.

Unfortunately, some practitioners cite reasons for failure before a technology is even considered or implemented because of poor funding or support for training. For sources of funding and ways to experiment with technology, visits to local or virtual assistive technology centers can be helpful. Practitioners can seek out conferences, local vendors, other AT users, and nearby AT centers to obtain information and training.

Other problems can arise as a student ages, or possibly when he or she becomes involved with different peer groups and feels different or singled out when using the technology. In contrast, with proper guidance and support, the same student can be made to feel special or be a model in the classroom. For example, through implementation of universal design, special software programs or devices no longer need to be used just by students with disabilities but should be made available for any students who need support or an alternative mode of learning. Precorrection to address this issue may simply rest in proper training for teachers and the student during implementation, with encouragement from general education teachers to continue the use of the technology across the curriculum.

As with any new topic, subject, or tool, students also need incentives and motivation to overcome barriers to learning. For students with mild disabilities, preconsideration of other nonacademic factors will provide additional assistance in the success of technology implementation. For example, Parette, Huer, and VanBiervliet (2005) discussed the effects of culture on technology implementation. Meskill and Hilliker (2005) discussed how factors related to family and community involvement may influence English language learners' use of technology. In the assessment process, factors like these need to be included 


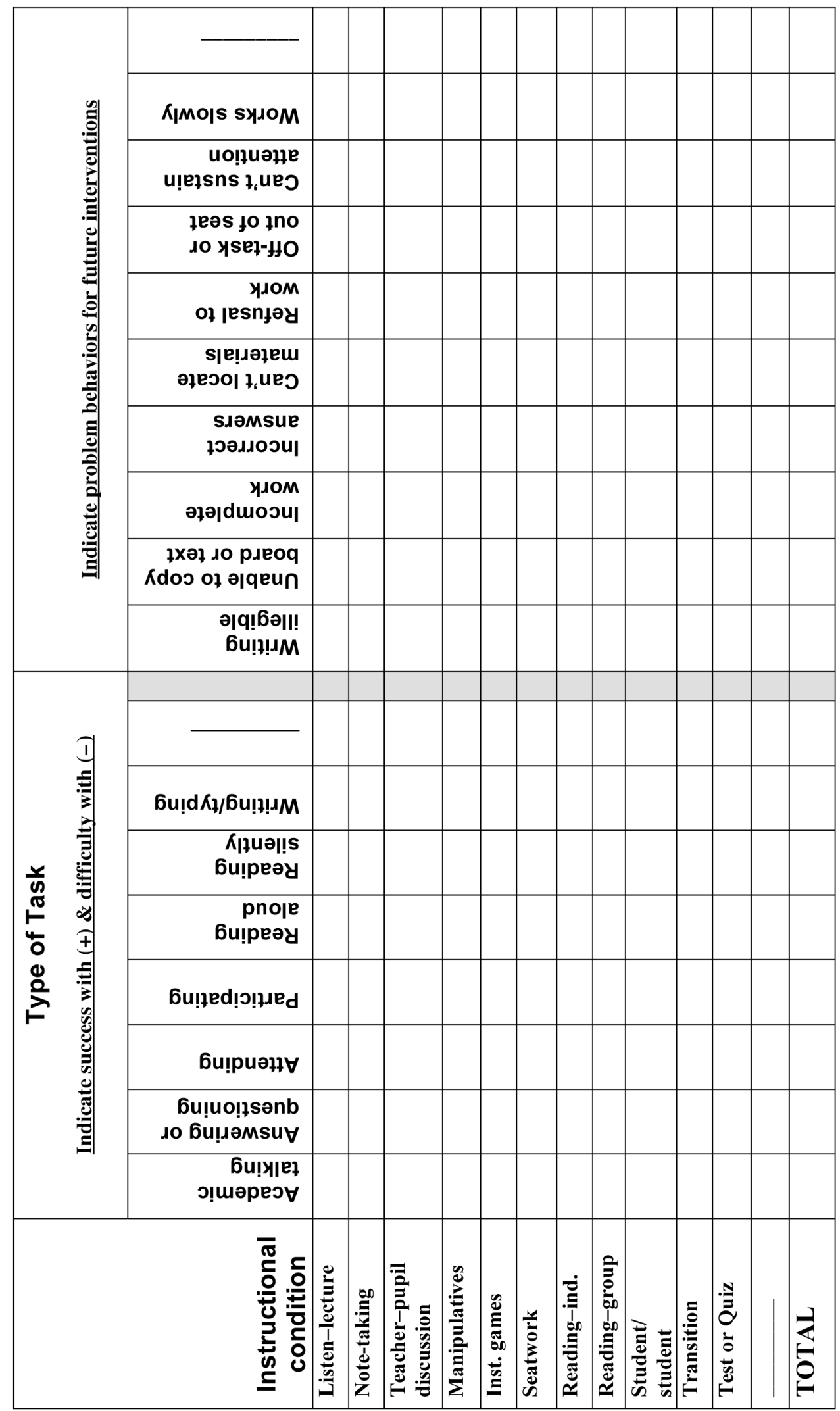


in the considerations at the onset as part of the context or environment.

An issue that may be more difficult to precorrect than other areas is the genuine lack of research on the success of specific technology applications to student characteristics. As Edyburn (2005) lamented, there are inadequate tools to support decision making in the selection of technology. Edyburn described this cautionary concern, distinguishing between remediation and compensation. At first, practitioners may focus on remediation and reteaching for the student. However, if we refer back to the FEAT model, we see that only emphasizing remediation options may result in limited or no success. For example, a teacher may strongly argue that students should to be able to complete all graphing functions using only paper-andpencil graphs with $100 \%$ accuracy before they are allowed to use a graphing calculator. In contrast, it might be significantly "worth it" to a student to use a graphing calculator $100 \%$ of the time in math class. If over time and different interventions a student still cannot perform a remedial task and as a consequence suffers repeated failures, it certainly seems logical that options involving compensatory technology must be considered. For teams that are evaluating AT for students with mild disabilities, a powerful and pragmatic level of competency may be knowing at what point it is best to conclude that remediation (technology based or otherwise) should be abandoned and technology-based compensatory solutions should be pursued to provide students with opportunities to experience success.

In choosing a specific technology, practitioners should rely on research-based information; however, sometimes no direct evidence base exists. Bausch (2006) described research indicating that students improve in their writing ability with auditory feedback and having their writing read aloud. We might subsequently translate this research to practice in our AT assessment model by considering the possibility of text-to-speech technology to help a student improve his or her writing. However, the use of this technology may still fail even if it is based on research, because some students may need to hear a human voice reading the written page rather than synthesized speech. Events like this need to be (a) documented in the progress-monitoring stages of AT assessment and implementation and (b) reported in some manner to the field. Researchers need information like this to make proper paradigm shifts in understanding the effects of AT tools and their implementation, and practitioners need to rely on current research to help make more informed assessment decisions.

In summary, technology applications for persons with disabilities can range from low- to high-tech software and assistive devices. For students with mild disabilities, technology needs can be considered across a variety of academic and environment settings and tasks, using a variety of systematic procedures and models of assessment. Practitioners and teachers need training on technology assessment and evaluation models, and access to these models and other supporting resources is available from many sources, some of which are free. When conducting assessment for technology, teams should always start with the student first and consider the skills that the student cannot perform and those that he or she wants to perform better. Goals must be set, and the student should be provided with direct instruction in the use of any technology tools that will be associated with meeting these goals. Effective technology assessment also includes progress monitoring. In order for technology implementation to be successful, data must be collected on discrete aspects of the student's work (e.g., quality, quantity, accuracy, speed, participation, spontaneity). Progress in the use of the technology tool must be monitored, and a reevaluation should be conducted if the student is not improving. In effect, assessment for technology is not really any different from highquality assessment methods and procedures that are used to prepare high-quality instruction.

\section{References}

Ashton, T. M. (2005). Students with learning disabilities using assistive technology in the inclusive classroom. In D. Edyburn, K. Higgins, \& R. Boone (Eds.), Handbook of special education technology research and practice (pp. 229-238). Whitefish Bay, WI: Knowledge by Design.

Assistive Technology Act of 1998, 29 U.S.C. $\$ 301$ et seq. (1998) Retrieved December 14, 2006, from http://www.section508 .gov/docs/AT1998.html

Assistive Technology Outcomes Measures (ATOMS). (2006). Informational database of assistive technology assessments. Milwaukee, WI: University of Milwaukee, Assistive Technology Outcomes Measurement System. Retrieved December 14, 2006, from http://www.r2d2.uwm.edu/atoms/idata

Baker, B. (1986). Using images to generate speech. In IEEE Biomedical Conference Proceedings. Fort Worth, TX: IEEE.

Ball, L. J., Bilyeu, D. V., Prentice, C., \& Beukelman, D. R. (2005). Augmentative and alternative communication: Infusing communication in an academic setting. In D. Edyburn, K. Higgins, \& R. Boone (Eds.), Handbook of special education technology research and practice (pp. 423-451). Whitefish Bay, WI: Knowledge by Design.

Bausch, M. (2006, September 6). Integrating research to practice. [Definition] Message posted to http://www.fctd.info/webboard/ archive.php .

Beukelman, D. R., \& Mirenda, P. (1998). Augmentative and alternative communication: Management of severe communication disorders in children and adults (2nd ed.). Baltimore: Brookes.

Blackhurst, A. E. (2001). A functional approach to the delivery of assistive technology services. Lexington: University of Kentucky, National Assistive Technology Research Institute. Retrieved 
December 14, 2006, from http://natri.uky.edu/resources/reports/ function.html

Bowser, G., \& Reed, P. (1998). Education tech points: A framework for assistive technology planning. Oshkosh: Wisconsin Assistive Technology Initiative. Retrieved December 14, 2006, from http://www.wati.org/AT_Services/edutech.html

Bowser, G., \& Reed, P. (2001). Hey, can I try that? A student handbook for choosing and using assistive technology. Oshkosh: Wisconsin Assistive Technology Initiative. Retrieved December 14, 2006, from http://www.wati.org/products/freematerials.html

Cook, A. M., \& Hussey, S. M. (2002). Assistive technology: Principles and practices (2nd ed.). St. Louis, MO: Mosby.

Coughenour, K. (2005). Teacher perceptions and use of accommodations in assessment and instruction. Unpublished dissertation, University of South Carolina, Columbia.

Edyburn, D. L. (2001). Models, theories, and frameworks: Contributions to understanding special education technology. Special Education Technology Practice. Retrieved October 12, 2006, from http://www.setp.net/pdf/4-2pp16-24.pdf

Edyburn, D. L. (2004). 2003 in review: A synthesis of the special education technology literature. Journal of Special Education Technology, 19(4), 57-80.

Edyburn, D. (2005). Assistive technology and students with mild disabilities: From consideration to outcome measurement. In D. Edyburn, K. Higgins, \& R. Boone (Eds.), Handbook of special education technology research and practice (pp. 229-238). Whitefish Bay, WI: Knowledge by Design.

Edyburn, D. L., \& Smith, R. O. (2004). Creating an assistive technology outcome measurement system: Validating the components. Assistive Technology Outcomes and Benefits, 1(1). Available at http://www.atia.org/atob/ATOBWeb/ATOBV1N1/index.htm

George, C. L., Schaff, J. I., \& Jeffs, T. L. (2005). Physical access in today's school: Empowerment through assistive technology. In D. Edyburn, K. Higgins, \& R. Boone (Eds.), Handbook of special education technology research and practice (pp. 355-377). Whitefish Bay, WI: Knowledge by Design.

Georgia Project for Assistive Technology (GPAT). (2006). Resources. Retrieved on December 14, 2006, from http:// www.gpat.org/Resources\%20Main.htm

Harris, J. (1997). Mining the Internet wetware: Why use activity structure? Learning and Leading with Technology, 25(4), 13-17.

Individuals with Disabilities Education Act of 1990, 20 U.S.C. $§ 1400$ et seq. (1990) (amended 1997).

Individuals with Disabilities Education Improvement Act of 2004, 20 U.S.C. $\$ 1400$ et seq. (2004) (reauthorization of the Individuals with Disabilities Education Act of 1990).

Johnstone, C. J., Altman, J., Thurlow, M. J., \& Thompson, S. J. (2006). A summary of research on the effects of test accommodations: 2002 through 2004 (Tech. Rep. No. 45). Minneapolis: University of Minnesota, National Center on Educational Outcomes. Retrieved December 14, 2006, from http://www.education.umn.edu/NCEO/ OnlinePubs/Tech45/default.html

King, T. W. (1999). Assistive technology: Essential human factors. Boston: Allyn \& Bacon.

Lahm, E. A., \& Case, D. (2003). UKAT toolkit. Lexington: University of Kentucky, Assistive Technology Project. Retrieved on December 14, 2006, from http://edsrc.coe.uky.edu/www/ukatii

Marino, M. T., Marino, E. C., \& Shaw, S. F. (2006). Making informed assistive technology decisions for students with high incidence disabilities. Teaching Exceptional Children, $38(6), 18-25$.
Meskill, C., \& Hilliker, S. (2005). English language learners and technology. In D. Edyburn, K. Higgins, \& R. Boone (Eds.), Handbook of special education technology research and practice (pp. 119-135). Whitefish Bay, WI: Knowledge by Design.

National Assistive Technology Research Institute. (2006). Assistive technology planner. Lexington: University of Kentucky, Author.

Okolo, C. M., Cavalier, A. R., Ferretti, R. P., \& MacArthur, C. A. (2000). Technology literacy and disabilities: A review of the research. In R. Gersten, E. P. Schiller, \& S. Vaughn (Eds.), Contemporary special education research: Syntheses of the knowledge base on critical instructional issues (pp. 179-250). Mahwah, NJ: Erlbaum.

O’Neill, R. E., Horner, R. H., Albin, R. W., Sprague, J. R., Story, K., \& Newton, J. S. (1997). Functional assessment of problem behavior: A practical handbook (2nd ed.). Pacific Grove, CA: Brooks/Cole.

Parette, H. P., Huer, M. B., \& VanBiervliet, A. (2005). Cultural research in special education technology. In D. Edyburn, K. Higgins, \& R. Boone (Eds.), Handbook of special education technology research and practice (pp. 81-103). Whitefish Bay, WI: Knowledge by Design.

Raskind, M. H., \& Bryant, B. R. (2002). FEAT_Functional evaluation for assistive technology. Austin, TX: Psycho-Educational Services.

Scherer, M. J. (1998). MPT assessments (1998 paper version). Webster, NY: The Institute for Matching Person and Technology, Inc. Retrieved December 12, 2006, from http://hometown.aol.com/ IMPT97/MPT.html

Scherer, M. J. (2005a). Assessing the benefits of using assistive technologies and other supports for thinking, remembering and learning. Disability and Rehabilitation, 27(13), 731-739.

Scherer, M. J. (2005b). MPT assessments: 2005 CD version. Webster, NY: The Institute for Matching Person and Technology, Inc. Retrieved December 12, 2006, from http://hometown.aol.com/ IMPT97/MPT.html

Smith, R. O. (2005). IMPACT2: Assistive technology outcomes measurement system. Milwaukee: University of Milwaukee. Retrieved December 14, 2006, from http://www.r2d2.uwm .edu/archive/impact2model.html

Texas Assistive Technology Network (TATN). (2006). Training modules online. Retrieved December 14, 2006, from http://www.texasat.net/default.aspx ?name=trainmod.home

Wissick, C. A. (2005a). Web toolboxes for educators: Free tools. Columbia: University of South Carolina. Retrieved December 12, 2006, from http://www.ed.sc.edu/caw/toolboxfree.html

Wissick, C. A. (2005b). Written language: When to consider technology. Technology in Action, Technology and Media Division, 1(6), 1-12.

Wissick, C. A., \& Schweder, W. (2006). Assistive technology centers: Getting technology into the hands of the users. Assistive Technology Associate Editor's Column. Journal of Special Education Technology, 21, 55-57.

Zabala, J. (1995). About the SETT framework. Lake Jackson, TX: Assistive Technology and Leadership. Retrieved December 14, 2006, from http://sweb.uky.edu/ jszaba0/JoySETT.html

Zabala, J. (2002). Update of the SETT framework. Lake Jackson, TX: Assistive Technology and Leadership. Retrieved December 14, 2006, from http://sweb.uky.edu/ jszaba0/SETTupdate2002.html

Zabala, J. S., Bowser, G., Blunt, M., Carl, D. F., Davis, S., Deterding, C., et al. (2000). Quality indicators for assistive technology services. Journal of Special Education Technology, 15(4), 25-36.

Zabala, J. S., \& Korsten, J. E. (1999). Assistive technology implementation and evaluation plan. Making a measurable difference with assistive technology: Evaluating the effectiveness of assistive 
technology. Lake Jackson, TX: Assistive Technology and Leadership. Retrieved December 14, 2006, from http://sweb .uky.edu/ jszaba0/ZabalaImplePlan2001.PDF

Cheryl A. Wissick, $\mathrm{PhD}$, is an associate professor of education in the Special Education Program at the University of South Carolina. Her current interests include assistive and instructional technology applications in special education, assessment, and working with students with learning disabilities.
J. Emmett Gardner, $\mathrm{PhD}$, is a professor of education in the Special Education Program at the University of Oklahoma. His current interests include assistive and instructional technology applications in special education, assessment, and working with students who have mild/moderate disabilities or are at risk of school failure. Address: Cheryl A. Wissick, 235 Wardlaw-Education, University of South Carolina, Columbia, South Carolina 29208. E-mail: cwissick@sc.edu 